\title{
Case Report: Human Monkeypox in the Kivus, a Conflict Region of the Democratic Republic of the Congo
}

\author{
Andrea M. McCollum,* Yoshinori Nakazawa, Guy Mutombo Ndongala, Elisabeth Pukuta, Stomy Karhemere, \\ Robert Shongo Lushima, Benoit Kebela Ilunga, Joelle Kabamba, Kimberly Wilkins, Jinxin Gao, Yu Li, Ginny Emerson, \\ Inger K. Damon, Darin S. Carroll, Mary G. Reynolds, Jean Malekani, and Jean-Jacques Muyembe Tamfum \\ Poxvirus and Rabies Branch, Division of High-Consequence Pathogens and Pathology, U.S. Centers for Disease Control and Prevention, Atlanta, \\ Georgia; Division Provinciale de la Santé, Ministère de la Santé Publique, Goma, Nord-Kivu, Democratic Republic of the Congo; \\ Institut National de Recherche Biomédicale, Kinshasa, Democratic Republic of the Congo; Ministère de la Santé Publique, \\ Kinshasa, Democratic Republic of the Congo; U.S. Centers for Disease Control and Prevention, Kinshasa, \\ Democratic Republic of the Congo; University of Kinshasa, Kinshasa, Democratic Republic of the Congo
}

\begin{abstract}
Monkeypox (MPX) is a zoonotic Orthopoxvirus infection endemic in central and western Africa. Human MPX cases occur in the central and northern regions of the Democratic Republic of the Congo (DRC), and this is the first report of confirmed MPX cases in the forested areas of North and South Kivu Provinces, with a detailed epidemiological investigation for one case. The location of each case is within areas predicted to be suitable for MPX virus transmission based on an ecological niche model. Phylogenetic analysis places these viruses in the Congo Basin clade.
\end{abstract}

\section{INTRODUCTION}

Monkeypox (MPX) is a zoonotic Orthopoxvirus (OPXV) infection. Symptoms include an initial febrile prodrome (14 days) followed by characteristic deep-seated, wellcircumscribed lesions typically present in a centrifugal distribution, including lesions on the palms of the hands and soles of the feet. Lesions on one area of the body are often synchronized in their development and in rash progression (macule, papule, vesicle, pustule, and crust) to desquamation, which occurs 14-24 days after rash onset. Lymphadenopathy is seen in many MPX patients but was not a common feature of smallpox. Transmission occurs via respiratory droplets or contact with infectious lesions (at all stages of the rash, including the crusts). Human-to-human transmission does occur and identification of individuals who have had significant contact with a MPX patient is important to limit the spread of disease. ${ }^{1}$ Prior smallpox vaccination (with the OPXV, vaccinia virus) confers protection against MPX virus (MPXV) infection. The average case fatality rate has been reported to be $11 \%$ among those without a prior smallpox vaccination. ${ }^{2}$

MPXV is endemic in areas of western and central Africa, with the overwhelming majority of reported cases from the Democratic Republic of the Congo (DRC). ${ }^{3}$ Within DRC, many cases are reported from the central and northern provinces, but very few cases are reported and investigated from the east, specifically in North and South Kivu. ${ }^{4}$ Previously, human MPX was confirmed from the "Kivu region" in Kibwe (Punia health zone) in 1982. Punia is located just west of North Kivu in what is now Maniema Province. This confirmed case of MPX in an infant resulted from the bite of a chimpanzee. ${ }^{5}$

\section{CASE REPORT}

The provinces of North and South Kivu have experienced a substantial amount of armed conflict in recent history. From 2011 through August 2014, health officials in North Kivu investigated four suspect MPX cases, and two suspect cases

*Address correspondence to Andrea M. McCollum, 1600 Clifton Road, MS A-30, Atlanta, GA 30333. E-mail: azv4@cdc.gov were investigated in South Kivu (Table 1); diagnostic specimens were submitted to the national laboratory. Two of the cases from North Kivu (Cases 1 and 2) and one case from South Kivu (Case 4) were positive for MPXV DNA signatures, one of the cases from North Kivu (Case 3) was positive for varicella zoster virus (VZV) DNA signatures, and one case each from North and South Kivu (Cases 5 and 6) had negative findings for both MPXV and VZV using polymerase chain reaction (PCR) diagnostic assays. Given the patients' ages, it was determined that none of the cases received a prior childhood smallpox vaccination.

A thorough clinical case report was available for Case 2, a 24-year-old male. Illness onset occurred while the patient was in the city of Butembo, on September 1 or 2 (see map, Figure 1). At that time, the patient initially visited the clinic at Nduko (Musienene health zone) for fever and rash. The patient described the onset of fever followed by a rash, starting on the face and mouth and then extending to the arms, trunk, and legs. On or about September 2, the patient was hospitalized at Nduko, where he received erythromycin, acetaminophen, promethazine, and vitamin $\mathrm{C}$. The presumptive diagnosis was VZV infection with suspicion of a human immunodeficiency virus (HIV) positive status (an HIV test was not conducted at this clinic). There was no change in the patient's condition after 7 days of hospitalization and he was transferred to the reference hospital in Katwa (Katwa health zone) on September 9. On admission to the hospital in Katwa, the patient was noted to have fever $\left(38^{\circ} \mathrm{C}\right)$, generalized rash, cough, and a swollen appearance to the face. The rash was described as the presence of umbilicated pustules surrounded by an area of erythema and also some crusts (yellow and black). An HIV test was performed and the result was negative. The local health staff were not sure of a presumptive diagnosis, and after local health authorities were consulted, suspect MPX infection was considered as a diagnosis. The patient was kept in isolation at the hospital and symptoms were treated with gentamicin, cloxacillin, narcotic analgesics, and a warm bath of permanganate. On September 11, the patient became afebrile and on September 12, it was noted that many skin lesions were evolving into crusts. On September 21, the patient was noted to have scars on the trunk, back, and hands and pustules on the thighs (Figure 2). 
TABLE 1

Characteristics of cases with specimens submitted for diagnostic testing for MPX from North and South Kivu

\begin{tabular}{|c|c|c|c|c|c|c|}
\hline Case & Province/health zone/village or town* & Gender, age (years) & Date of illness onset $\dagger$ & Date of specimen collection, type of specimen & OPXV/MPXV PCR & VZV PCR \\
\hline 1 & North Kivu/Walikale/Boboro & Male, 28 & November 22, 2011 & $\begin{array}{l}\text { November } 23 \text {, vesicular swab } \\
\text { and blood }\end{array}$ & Positive & N/A \\
\hline 2 & North Kivu/Musienene/Butembo & Male, 24 & September 1 or 2,2012 & September 12 , crust and blood & Positive & N/A \\
\hline 3 & North Kivu/Kayna/Kaleko & Female, 1.6 & December 1, 2012 & $\begin{array}{l}\text { December 4, 2012, blood; } \\
\text { December 8, 2012, } \\
\text { vesicular swab and crust }\end{array}$ & Negative & Positive \\
\hline 4 & South Kivu/Shabunda/Mabaka & Female, 23 & December 8, 2012 & December 17 , blood & Positive & N/A \\
\hline 5 & North Kivu/Butembo/Butembo & Male, 8 & Unknown & July 19 , blood & Negative & Negative \\
\hline 6 & South Kivu/Minova/Bunge-Zicano & Male, 1.5 & May 19, 2014 & May 29, blood & Negative & Negative \\
\hline
\end{tabular}

MPXV = monkeypox virus; $\mathrm{N} / \mathrm{A}$ = not applicable; OPXV = Orthopoxvirus, $\mathrm{PCR}$ = polymerase chain reaction; $\mathrm{VZV}=$ varicella zoster virus

*The locality information describes where the case lived at the time of illness onset.

$\dagger$ Signs and symptoms present at the time of illness onset (i.e., fever, rash) are not precisely known.

$\ddagger$ Suspect cases were reclassified as confirmed MPX cases if an original specimen tested positive for Orthopoxvirus (OPXV) DNA signatures at Institut National de Recherche Biomedicale $(\text { INRB })^{6}$ and/or if a specimen tested positive for MPXV DNA signatures at U.S. Centers for Disease Control and Prevention (CDC). ${ }^{7,8}$ Cases were classified as confirmed VZV cases if a specimen tested positive for VZV DNA signatures at INRB (reagents provided by the U.S. Army Medical Research Institute of Infectious Diseases, unpublished protocol).

On September 20, an epidemiological investigation was launched by local health authorities to identify additional cases in the community, ascertainment of contacts and potential health-care-associated exposure, and strengthen local surveillance for disease. The investigation identified 30 contacts while the patient was ill, including family members and health-care workers. Of those, $23(76.7 \%)$ were health-care workers at the clinic in Nduko or at the hospital in Katwa. Among all contacts, 16 (53\%) had no prior vaccination against smallpox (assessed by visual examination of a smallpox vaccination scar), including $14(60.9 \%)$ of the health-care workers. Contacts were followed for 21 days after the date of last contact with Case 2. None of the contacts became ill and no additional cases were reported from neighboring health zones.

The patient was a farmer and had recently traveled from a highly forested area (Mapendano in the health zone of Manguredjipa) on August 28. He reported traveling $>200 \mathrm{~km}$ to the Butembo area, arriving sometime between August 28 and September 1, and illness onset occurred after his arrival in Butembo. Given the average incubation period of human MPX (7-17 days), it is likely that Case 2 acquired the virus in Mapendano, before his voyage. The patient reported that he had handled monkeys killed by local hunters, saved monkey meat for his voyage, and then ate this meat over the course of his travel. Case 1 also noted contact with bushmeat before illness onset.

Each of the three confirmed MPX cases from the Kivus had traveled from or presented to clinics in the westernmost, highly forested areas of the provinces (Figure 1). The location of each case was within areas predicted to be suitable for MPXV transmission based on an ecological niche model developed to predict the range of MPXV using known ecological and environmental variables and confirmed cases of human disease. ${ }^{9}$ This model was created with the Maxent algorithm ${ }^{10,11}$ and predicts suitable environmental areas for disease transmission across central and northern DRC, extending through the westernmost portion of the Kivus. The predictions limit the area of risk to the region west of the Albertine Rift and associated mountain ranges in the Kivus. Confirmation of these three cases lends further support to this model of disease occurrence.

DNA was isolated from diagnostic specimens collected from Cases 1, 2, and 4, sequenced using Illumina (San Diego, CA) paired-end sequencing and assembled using CLC Genomics Workbench 7.0.3 (http://www.clcbio.com). Five genes from the central coding region corresponding to the $A 10 L, A 24 R, D 1 R$,
$E 9 L$, and $J 6 R$ genes in cowpox virus were used to estimate a maximum probability tree in the Bayes module of Geneious 6.1.2 (http://www.geneious.com). ${ }^{12,13}$ These five genes are present in a conserved region of the genome, previously used to explore relationships between OPXVs. ${ }^{14}$ For this analysis, we extracted the same genes from 35 MPX genomes published in GenBank and included two isolates as outgroups (cowpox virus: X94355.2 and horsepox virus: DQ792504.1). Figure 3 shows the phylogenetic tree in which these cases are shown

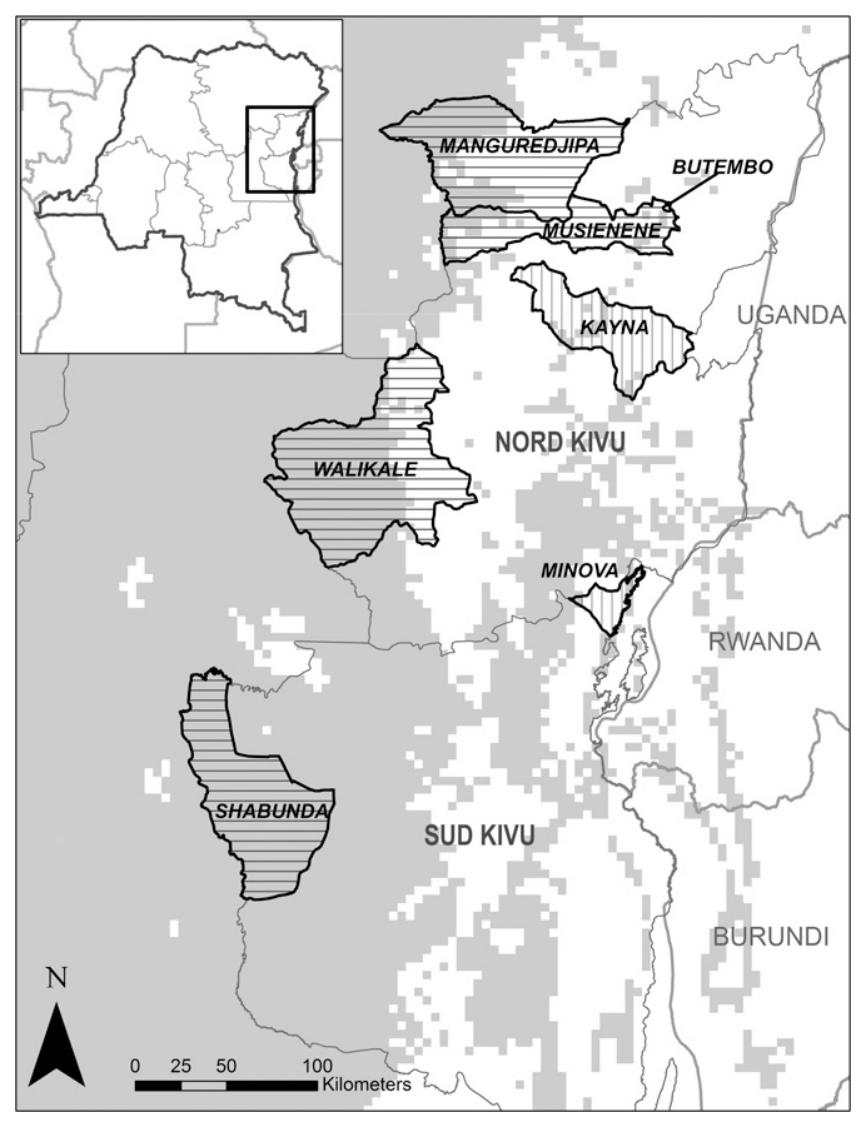

FIGURE 1. Map showing the health zones for the six investigated cases: Walikale (Case 1), Musienene/Manguredjipa (Case 2), Kayna (Case 3), Mabaka (Case 4), Butembo (Case 5), and Minova (Case 6). Horizontal hatched lines represent health zones with a confirmed monkeypox (MPX) case and vertical hatched lines represent health zones with cases confirmed to not be MPX cases. Predicted suitable areas of transmission from Maxent model are shown in gray. 



FIgURE 2. Photographs of Case 2 (dated assuming illness onset occurred on September 1). (A) 10 days after illness onset, pustules on the face and chest; (B) 13 days after illness onset, pustules on the palms of the hands; (C) 20 days after illness onset, scarring and hypopigmentation on the back after separation of crusts; and (D) 20 days after illness onset, pustules on the left foot.

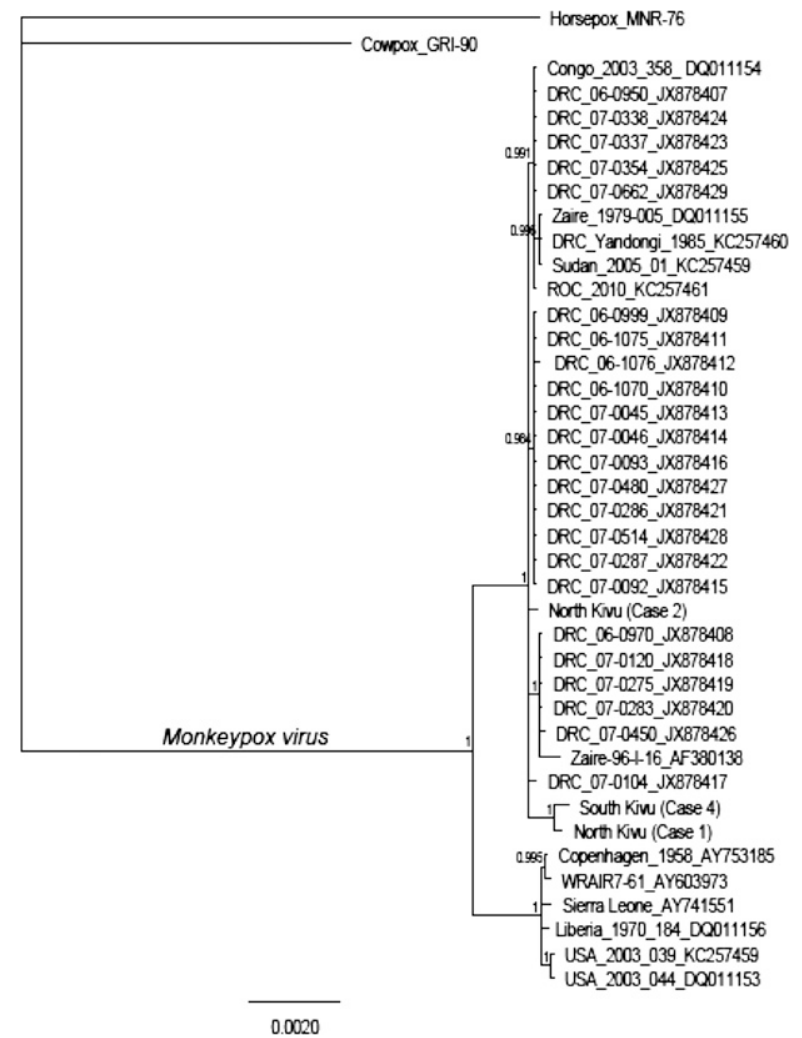

Figure 3. Phylogram based on the five selected genes ( $A 10 L$, $A 24 R, D 1 R, E 9 L$, and $J 6 R)$. The three cases from the Kivus are included within the Congo Basin monkeypox virus (MPXV) clade. Clade credibility values are shown at nodes. Scale bar indicates expected substitutions per site. (GenBank accession numbers: I37_A10L KP739434, I37_A24R KP739435, I37_D1R KP739436, I37_E9L KP739437, I37_J6R KP739438, I38_A10L KP739439, I38_A24R KP739440, I38_D1R KP739441, I38_E9L KP739442, I38_J6R KP739443，I14_A10L KP739444, I14_A24R KP739445, I14_D1R KP739446, I14_E9L KP739447, I14_J6R KP739448.) in relation to other already published isolates based on an analysis of the five selected genes. Although the relationships are not completely resolved, posterior probabilities support the inclusion of the Kivu isolates in the Congo Basin clade. In addition, the analysis suggests that at least two of the viruses from the Kivu area (Cases 1 and 4) may share some genetic traits that differentiate them from the rest of the Congo Basin isolates.

\section{DISCUSSION}

Reports from Case 2 indicates that MPX suspicion developed after consultation with additional health authorities; the clinicians and nurses treating the patient may not have initially considered this as a possibility. Reports of MPX are sparse from the Kivus, and few cases of suspect MPX are investigated with diagnostic specimens collected; only six such cases were investigated in 3.5 years. Health-care workers at the periphery of zones at risk of MPX should be educated on the risk of disease, and recognition, treatment, and isolation of cases. Thorough follow-up of suspect cases and their contacts (social, familial, and health care associated) is warranted to prevent further transmission of the virus. Investigation and confirmation of suspect cases will be critical for further understanding of the virus' range in DRC and help bolster surveillance activities in these areas.

The Kivu region is an area that has experienced armed conflict and population displacements for almost 20 years. In 2012, a new wave of fighting accelerated the movement of people and there were an estimated 490,000 people displaced as a result of conflict in the Kivus. ${ }^{15}$ Movement of people to less conflicted and more forested areas imparts a greater risk of zoonotic infections such as MPX. ${ }^{16}$ Further, smallpox vaccination confers protection against other OPXVs, including MPXV, but routine vaccination was discontinued in DRC in 1982 after smallpox eradication. There are an increasing 
number of people who never received vaccine-derived immunity to OPXVs, and this has been posited as a reason for an increase in the number of MPX cases in another area of DRC since the period of smallpox eradication. ${ }^{17}$ The movement of individuals into the forests and the lack of vaccine-derived immunity places a larger number of people at risk of acquiring MPXV and developing disease.

Received February 2, 2015. Accepted for publication March 31, 2015.

Published online August 17, 2015.

Acknowledgments: We greatly appreciate the assistance of local health-care workers in the investigations.

Disclaimer: The findings and conclusions in this report are those of the author(s) and do not necessarily represent the views of the Centers for Disease Control and Prevention.

Authors' addresses: Andrea M. McCollum, Yoshinori Nakazawa, Kimberly Wilkins, Jinxin Gao, Yu Li, Ginny Emerson, Inger K. Damon, Darin S. Carroll, and Mary G. Reynolds, Poxvirus and Rabies Branch, U.S. Centers for Disease Control and Prevention, Atlanta, GA, E-mails: azv4@cdc.gov, inp7@cdc.gov, ibx4@cdc.gov, wzo5@cdc.gov, dtt4@cdc.gov, iad7@cdc.gov, zuz4@cdc.gov, and nzr6@ cdc.gov. Guy Mutombo Ndongala, Division Provinciale de la Santé, Ministère de la Santé Publique, Goma, Nord-Kivu, Democratic Republic of the Congo, E-mail: guymutombon@yahoo.fr. Elisabeth Pukuta, Stomy Karhemere, and Jean-Jacques Muyembe Tamfum, Institut National de Recherche Biomédicale, Kinshasa, Democratic Republic of the Congo, E-mails: elisepukuta@gmail.com, stomy_ karhem@yahoo.fr, and muyembejj@gmail.com. Robert Shongo Lushima and Benoit Kebela Ilunga, Ministère de la Santé Publique, Kinshasa, Democratic Republic of the Congo, E-mails: robert_ shongo@yahoo.fr and kebelailunga@yahoo.fr. Joelle Kabamba, U.S. Centers for Disease Control and Prevention, Kinshasa, Democratic Republic of the Congo, E-mail: jlz7@cdc.gov. Jean Malekani, University of Kinshasa, Kinshasa, Democratic Republic of the Congo, E-mail: elevagefaune@yahoo.fr.

\section{REFERENCES}

1. Di Giulio DB, Eckburg PB, 2004. Human monkeypox: an emerging zoonosis. Lancet Infect Dis 4: 15-25.

2. Jezek Z, Szczeniowski M, Paluku KM, Mutombo M, 1987. Human monkeypox: clinical features of 282 patients. J Infect Dis 156: 293-298.

3. Reynolds MG, Damon IK, 2012. Outbreaks of human monkeypox after cessation of smallpox vaccination. Trends Microbiol 20: $80-87$.

4. Rimoin AW, Kisalu N, Kebela-Ilunga B, Mukaba T, Wright LL, Formenty P, Wolfe ND, Shongo RL, Tshioko F, Okitolonda E, Muyembe JJ, Ryder R, Meyer H, 2007. Endemic human monkeypox, Democratic Republic of Congo, 2001-2004. Emerg Infect Dis 13: 934-937.
5. Mutombo M, Arita I, Jezek Z, 1983. Human monkeypox transmitted by a chimpanzee in a tropical rain-forest area of Zaire. Lancet 1: 735-737.

6. Kulesh DA, Baker RO, Loveless BM, Norwood D, Zwiers SH, Mucker E, Hartmann C, Herrera R, Miller D, Christensen D, Wasieloski LP Jr., Huggins J, Jahrling PB, 2004. Smallpox and pan-orthopox virus detection by real-time $3^{\prime}$-minor groove binder TaqMan assays on the roche LightCycler and the Cepheid smart Cycler platforms. J Clin Microbiol 42: 601-609.

7. Li Y, Olson VA, Laue T, Laker MT, Damon IK, 2006. Detection of monkeypox virus with real-time PCR assays. J Clin Virol 36: 194-203.

8. Li Y, Zhao H, Wilkins K, Hughes C, Damon IK, 2010. Real-time PCR assays for the specific detection of monkeypox virus west African and Congo Basin strain DNA. J Virol Methods 169: 223-227.

9. Nakazawa Y, Emerson GL, Carroll DS, Zhao H, Li Y, Reynolds MG, Karem KL, Olson VA, Lash RR, Davidson WB, Smith SK, Levine RS, Regnery RL, Sammons SA, Frace MA, Mutasim EM, Karsani ME, Muntasir MO, Babiker AA, Opoka L, Chowdhary V, Damon IK, 2013. Phylogenetic and ecologic perspectives of a monkeypox outbreak, southern Sudan, 2005. Emerg Infect Dis 19: 237-245.

10. Phillips SJ, Anderson RP, Schapire RE, 2006. Maximum entropy modeling of species geographic distributions. Ecol Modell 190: 231-259.

11. Phillips SJ, Dudik M, Schapire RE, 2004. A maximum entropy approach to species distribution modeling. Proceedings of the Twenty-First International Conference on Machine Learning. $655-662$.

12. Huelsenbeck JP, Ronquist F, 2001. MRBAYES: Bayesian inference of phylogenetic trees. Bioinformatics 17: 754-755.

13. Kearse M, Moir R, Wilson A, Stones-Havas S, Cheung M, Sturrock S, Buxton S, Cooper A, Markowitz S, Duran C, Thierer T, Ashton B, Meintjes P, Drummond A, 2012. Geneious Basic: an integrated and extendable desktop software platform for the organization and analysis of sequence data. Bioinformatics 28: 1647-1649.

14. Emerson GL, Li Y, Frace MA, Olsen-Rasmussen MA, Khristova ML, Govil D, Sammons SA, Regnery RL, Karem KL, Damon IK, Carroll DS, 2009. The phylogenetics and ecology of the orthopoxviruses endemic to North America. PLoS One 4: e7666.

15. UNHCR, The UN Refugee Agency, 2013. Emergency Response for the Situation in the Eastern Democratic Republic of the Congo.

16. Reynolds MG, Emerson GL, Pukuta E, Karhemere S, Muyembe JJ, Bikindou A, McCollum AM, Moses C, Wilkins K, Zhao H, Damon IK, Karem KL, Li Y, Carroll DS, Mombouli JV, 2013. Detection of human monkeypox in the Republic of the Congo following intensive community education. Am J Trop Med Hyg 88: 982-985.

17. Rimoin AW, Mulembakani PM, Johnston SC, Lloyd Smith JO, Kisalu NK, Kinkela TL, Blumberg S, Thomassen HA, Pike BL, Fair JN, Wolfe ND, Shongo RL, Graham BS, Formenty P, Okitolonda E, Hensley LE, Meyer H, Wright LL, Muyembe JJ, 2010. Major increase in human monkeypox incidence 30 years after smallpox vaccination campaigns cease in the Democratic Republic of Congo. Proc Natl Acad Sci USA 107: $16262-16267$. 